\title{
THE CONNECTION BETWEEN ALZHEIMER'S DISEASE AND DIABETES
}

\author{
A. M. Ciobanu ${ }^{1}$, C. Neagu², L. M. Catrinescu² \\ ${ }^{1}$ Associate professor, PhD; University of Medicine and Pharmacy "Carol Davila" Bucharest, \\ department of psychiatry, head of $1^{\text {st }}$ department at "Alexandru Obregia" Clinical Hospital of Psychiatry, Bucharest \\ ${ }^{2}$ Resident doctor, $1^{\text {st }}$ department at "Alexandru Obregia" Clinical Hospital of Psychiatry, Bucharest
}

\section{OBJECTIVES}

Our main purpose is to create a meta-analysis of existent studies and articles from medical literature to verify our assumptions that Alzheimer's disease is the new type of diabetes, also known as type 3 diabetes.

\section{BACKGROUND AND AIMS}

Diabetes and Alzheimer's appear to be two distinct diseases. However, both conditions involve impaired glucose homeostasis and altered brain function.

Both Alzheimer's disease and type 2 diabetes are characterized by a genetic predisposition, increased prevalence with aging and comparable pathological features in the islet and brain (amyloid derived from amyloid $\beta$ protein in the brain in Alzheimer's disease and islet amyloid derived from islet amyloid polypeptide in the pancreas in type 2 diabetes) $)^{2}$.

In Alzheimer's disease there is a decreased glucose utilization and studies have shown that insulin treatment has improved memory, which implies that there is an association between insulin and cognition.

Studies mention "glycotoxic" Alzheimer's disease in which inflammation from high blood glucose levels is combined with a trophic loss of insulin sensitivity. Research demonstrates that blood sugar dysregulation is linked to neurodegeneration, thus supporting the concept of glycotoxic Alzheimer's disease ${ }^{1}$.

While the main function of insulin is to regulate blood sugar, it also is thought to play a role in Alzheimer's disease pathogenesis, having shown reduced levels of insulin and insulin signaling in patients' brains. Moreover, an interrelation between these entities is suggested by: increased risk of developing minor cognitive deficits, dementia or Alzheimer's disease by patients with Type 1 or 2 Diabetes, obesity or dyslipidemia; progressive insulin resistance of the brain or even its deficiency in patients diagnosed with Alzheimer's Disease or minor cognitive deficits after treatment with insulin sensitizing agents ${ }^{4}$.

\section{MATERIALS AND METHODS}

We compared studies and articles in the literature that highlight the link between diabetes and Alzheimer's disease. The analysis was performed by Google academic and PubMed. We analyzed the results of over 30 publications from 2005 to 2016 in which we identified the common mechanisms of these pathologies ${ }^{3}$.

\section{Inclusion criteria:}

- Population, longitudinal studies examining the relationship be tween diabetes and Alzheimer's disease using:

- patients diagnosed with Alzheimer's Disease (DSM4 criteria); - patients diagnosed with diabetes 1 or 2;
- Scientific articles analyzing dysfunctions in glucose metabolism in patients with Alzheimer's disease.

\section{Exclusion criteria:}

- Studies in which patients had multiple comorbidities.

- Studies whose inclusion criteria are not superimposed.

\section{RESULTS}

Neuroimaging studies have shown that diabetic patients have moderate-high risk of developing lacunae, hippocampal atrophy and white matter lesions.

So, we can expect an increased risk of developing vascular disease in these patients ${ }^{1}$

Following systematic analysis of population studies, patients with a diabetes diagnosis also had a significantly increased incidence of Alzheimer's disease compared to those without this diagnosis.

With the availability of treatment options for managing diabetes and its complications, the life expectancy of a person diagnosed with diabetes is increasing ${ }^{5}$. The association between diabetes and AD is likely to be more evident in the future rather than with the current older population because of survival bias. Health care systems will be facing a growing population worldwide of patients with $A D$ and diabetes ${ }^{6}$.

In the elderly diabetic patients cognitive disfunction makes it difficult for them to perform tasks including cognitive tests, glucose monitoring, adjusting the time and dose of insulin.

Studies examining the chronic neurodegenerative pathways in patients with with diabetes can lead to development of possible treatments that delay cognitive decline and improve the quality of life, therefore, current antidiabetic medication with neuroprotective action need to be given serious considerations.

\section{CONCLUSIONS}

Diabetes and Alzheimer's disease have been thought to be two independent disorders. However, the results of epidemiological investigations have suggested possible associations or some common mechanisms. If true, common pharmacotherapy should be an effective treatment.

Clinical practice has shown that there may be a steady or even favorable evolution of Alzheimer's disease, especially under rigorous control of glucose level in patients diagnosed with type 1 or 2 diabetes.

By optimizing the control of blood glucose, we are protecting the health of the brain and maybe even prevent the irreversible cognitive changes that appear in Alzheimer's disease. Since insulin plays a key role in cognitive processes and synaptic plasticity, boosting this type of signaling may come to be a therapeutic approach in AD.

\section{BIBLIOGRAPHY}

1 - „Alzheimer's disease and type 2 diabetes: multiple mechanisms contribute to interactions”Jayaraman, Anusha and Christian $\mathrm{J}$ Pike. Current diabetes reports vol. 14,4 (2014): 476.

2 - „Diabetes Mellitus and the risk of dementia- a population based study”, Weil Xu, Karoliska Institute, Stockholm, Sweden, 2008;

3 - ,,The relationship between Alzheimer's disease and diabetes”: Type 3 diabetes?, Zina Kroner, Alternative medicine review, Volume 4 . Nr. 4, 2009;

4 - , Insulin resistance and Alzheimer's disease”, Suzanne M. de la Monte, BMB Rep. 2009 Aug 31; 42(8): 475-481) ;

5 - S. loacara, C. Guja, C. lonescu-Tirgoviste, S. Fica, S. Sabau, S. Radu, A. Micu, C. Tiu Improvements in life expectancy in adult type 2 diabetes patients in the last six decades Diabetes Res. Clin. Pract., 92 (2011), pp. 400-404

6 - W. Han, C. Li, Linking type 2 diabetes and Alzheimer's disease Proc. Natl. Acad. Sci. U. S. A., 107 (2010), pp. $6557-6558$ 